\title{
Effects of natural sounds on the perception of road traffic noise
}

Bert De Coensel, Sofie Vanwetswinkel, and Dick Botteldooren

Citation: The Journal of the Acoustical Society of America 129, EL148 (2011); doi: 10.1121/1.3567073

View online: https://doi.org/10.1121/1.3567073

View Table of Contents: https://asa.scitation.org/toc/jas/129/4

Published by the Acoustical Society of America

\section{ARTICLES YOU MAY BE INTERESTED IN}

Perceptual assessment of quality of urban soundscapes with combined noise sources and water sounds The Journal of the Acoustical Society of America 127, 1357 (2010); https://doi.org/10.1121/1.3298437

Acoustical characteristics of water sounds for soundscape enhancement in urban open spaces

The Journal of the Acoustical Society of America 131, 2101 (2012); https://doi.org/10.1121/1.3681938

Acoustical and perceptual assessment of water sounds and their use over road traffic noise

The Journal of the Acoustical Society of America 133, 227 (2013); https://doi.org/10.1121/1.4770242

A principal components model of soundscape perception

The Journal of the Acoustical Society of America 128, 2836 (2010); https://doi.org/10.1121/1.3493436

Designing sound and visual components for enhancement of urban soundscapes

The Journal of the Acoustical Society of America 134, 2026 (2013); https://doi.org/10.1121/1.4817924

Similarity and pleasantness assessments of water-fountain sounds recorded in urban public spaces

The Journal of the Acoustical Society of America 138, 3043 (2015); https://doi.org/10.1121/1.4934956

\section{Advance your science and career as a member of the}




\title{
Effects of natural sounds on the perception of road traffic noise
}

\author{
Bert De Coensel, ${ }^{\text {a) }}$ Sofie Vanwetswinkel, and Dick Botteldooren \\ Acoustics Group, Department of Information Technology, Ghent University, \\ St.-Pietersnieuwstraat 41, B-9000 Ghent, Belgium \\ bert.decoensel@intec.ugent.be,sofie.vanwetswinkel@ugent.be, \\ dick.botteldooren@intec.ugent.be
}

\begin{abstract}
Recent studies show that introducing sound from water features in urban open spaces may reduce the loudness of road traffic noise, but it is not clear in which situations this measure also improves overall soundscape quality. This work describes a listening experiment on loudness, pleasantness, and eventfulness of stimuli that combine road traffic noise with fountain or bird sound at different sound levels. Adding fountain sound reduced the loudness of road traffic noise only if the latter had low temporal variability. Conversely, adding bird sound significantly enhanced soundscape pleasantness and eventfulness, more than what was achieved by adding fountain sound.
\end{abstract}

(C) 2011 Acoustical Society of America

PACS numbers: 43.50.Rq, 43.50.Qp, 43.66.Lj, 43.66.Ba [MS]

Date Received: January 21, 2011 Date Accepted: February 22, 2011

\section{Introduction}

Reducing road traffic noise in urban environments is often not feasible due to economical or mobility reasons. An alternative approach to improve the quality of the soundscape in well defined spaces such as urban parks, which has gained scholarly interest, is to introduce wanted sounds to mask or distract attention away from unwanted sounds. ${ }^{1,2}$ From perceptual tests, it was found that the sound of water features (streams, fountains, etc.) would be the most effective natural sound to mask road traffic noise. ${ }^{3}$ For this purpose, the acoustical properties of water features have been studied by several researchers recently. ${ }^{4,5}$ In most cases, it will not be possible to energetically mask the noise from road traffic, even if the water features produce relatively high sound levels. ${ }^{4,6}$ On the other hand, introducing sound from water features in the urban environment may still reduce the loudness of road traffic noise ${ }^{1}$ due to informational masking effects such as target-masker confusion. ${ }^{7}$ However, it is not clear in which situation the addition of sounds also improves the overall soundscape quality. Next to this, most studies only considered the road traffic noise with small fluctuations in sound level, ${ }^{1,3,5}$ which is not always realistic for urban environments.

This paper describes a listening experiment in which binaural recordings of different types of road traffic noise are combined with the sound of a fountain and of birds at different sound levels. Participants have to assess the loudness of the road traffic noise within the various stimuli, using a paired comparison test, and the pleasantness and eventfulness of the overall stimuli, using a semantic differential test. The purpose of the listening experiment was (i) to explore the potential of fountain and bird sound for reducing the perceived loudness of road traffic noise with time-varying as well as with stationary sound pressure level and (ii) to find out if a reduction in the loudness of road traffic noise, caused by introducing fountain or bird sound, leads to an improved overall soundscape quality.

\footnotetext{
${ }^{a)}$ Author to whom correspondence should be addressed.
} 
Table 1. Composition and acoustical parameters of the stimuli (energetic average between left and right ear) together with the BTL scale values of the paired comparison test (loudness of road traffic noise) and the aggregated results of the semantic differential test (pleasantness and eventfulness of overall soundscape).

\begin{tabular}{|c|c|c|c|c|c|c|c|c|c|}
\hline \multirow[b]{2}{*}{ Label } & \multirow[b]{2}{*}{ Composition } & \multicolumn{5}{|c|}{ Levels [dB(A)] } & \multirow{2}{*}{$\frac{\text { BTL scale }}{\text { Loudness }}$} & \multicolumn{2}{|c|}{ Semantic differential } \\
\hline & & $L_{\mathrm{Aeq}}$ & SNR & $L_{\mathrm{A} 10}$ & $L_{\mathrm{A} 10}-L_{\mathrm{A} 90}$ & $L_{\mathrm{Ceq}}-L_{\mathrm{Aeq}}$ & & Pleasantness & Eventfulness \\
\hline FwO & Freeway & 65.8 & - & 66.8 & 2.1 & 8.7 & 4.11 & 2.14 & 1.19 \\
\hline FwB1 & +Birds & 66.1 & -11.9 & 67.0 & 2.1 & 8.4 & 3.35 & 2.78 & 2.66 \\
\hline FwB2 & - & 68.9 & 0.1 & 72.2 & 7.0 & 6.0 & 3.02 & 3.34 & 3.47 \\
\hline FwF1 & + Fountain & 65.9 & -14.9 & 66.9 & 2.0 & 8.6 & 2.88 & 2.23 & 1.48 \\
\hline FwF2 & - & 67.1 & -4.5 & 67.9 & 1.6 & 7.5 & 2.83 & 2.56 & 1.89 \\
\hline $\mathrm{MaO}$ & Major road & 62.6 & - & 65.5 & 8.2 & 6.7 & 2.18 & 2.60 & 4.59 \\
\hline MaB1 & +Birds & 63.4 & -9.2 & 66.1 & 7.5 & 6.1 & 2.23 & 4.16 & 5.61 \\
\hline MaB2 & - & 73.8 & 10.8 & 77.5 & 13.3 & 0.1 & 2.30 & 4.37 & 6.21 \\
\hline MaF1 & + Fountain & 63.0 & -10.0 & 65.7 & 7.3 & 6.4 & 1.68 & 3.57 & 4.90 \\
\hline MaF2 & - & 65.0 & -1.5 & 66.9 & 4.5 & 4.9 & 1.29 & 3.41 & 4.45 \\
\hline $\mathrm{MiO}$ & Minor road & 59.6 & - & 63.1 & 11.9 & 7.1 & 1.23 & 4.49 & 6.44 \\
\hline MiB1 & + Birds & 60.1 & -9.3 & 63.2 & 10.9 & 6.7 & 1.00 & 5.19 & 6.70 \\
\hline MiB2 & - & 71.6 & 11.7 & 75.7 & 14.9 & -0.1 & 1.28 & 5.88 & 7.28 \\
\hline MiF1 & + Fountain & 60.0 & -10.5 & 63.2 & 9.8 & 6.8 & 1.15 & 4.85 & 6.10 \\
\hline MiF2 & - & 61.9 & -1.5 & 64.1 & 5.3 & 5.3 & 1.43 & 4.62 & 5.62 \\
\hline
\end{tabular}

\section{Methodology}

\subsection{Participants}

One hundred subjects (45 females, 55 males) participated in a 1-h laboratory session. The mean age of the subjects was $28.6 \mathrm{yr}$ (standard deviation $=10.4$; range $=18-62$ ). After the experiment, a small questionnaire was administered, which contained questions on self-assessed impaired hearing; all subjects reported normal hearing ability. The participants received a cinema ticket as compensation.

\subsection{Stimuli}

Binaural recordings of road traffic noise were made using a $\mathrm{B} \& \mathrm{~K}$ head and torso simulator (HATS) type 4128C (Bruel \& Kjaer, Denmark) near three types of road: the E40 freeway in Ghent (vehicle speed of $120 \mathrm{~km} / \mathrm{h}, 2 \times 3$ lanes, traffic intensity of 3700 vehicles/h in each direction during daytime) at a distance of $150 \mathrm{~m}$ from the closest lane; the Bredabaan, a major road in Antwerp $(70 \mathrm{~km} / \mathrm{h}, 2 \times 2$ lanes, 600 vehicles $/ \mathrm{h})$ at $80 \mathrm{~m}$; and the Waterstraat, a minor road in Antwerp $(50 \mathrm{~km} / \mathrm{h}, 2 \times 1$ lane, 100 vehicles $/ \mathrm{h}$ ) at $60 \mathrm{~m}$. Three representative excerpts which contained only the sound from road traffic were selected, one for each type of road. Table 1 shows the acoustical parameters of the selected road traffic noise excerpts (labeled "xxO"). Individual passages of cars can easily be discerned from the noise of the minor urban road, while this is not possible for the noise of the freeway. The noise of the major urban road represents the intermediate case in which individual passages can be discerned only when auditory attention is focused on the sound. This is reflected in the values of $L_{\mathrm{A} 10}-L_{\mathrm{A} 90}$, which measures the temporal variability of the sounds, ranging from $2.1 \mathrm{~dB}(\mathrm{~A})$ for the freeway noise to $11.9 \mathrm{~dB}(\mathrm{~A})$ for the minor road noise.

Binaural recordings of fountain sound were made at varying distances (1-20 $\mathrm{m}$ ) from a fountain, located on a square in Ghent that is enclosed by buildings on all sides, and without roads. The fountain consisted of water falling along all sides of a rectangular stone column of height $2 \mathrm{~m}$, standing inside a pool with a depth of about $50 \mathrm{~cm}$, producing a constant water sound. Binaural recordings of the sound of birds singing were created by placing the HATS in an anechoic chamber, and by playing 
back bird sound from a compact disc (CD) (monaural) through a single loudspeaker placed in front of the HATS. This procedure was used in order to make sure no other disturbing sounds were present in the binaural recordings. Six representative excerpts of fountain sound and six excerpts of bird sound were selected, which had A-weighted sound pressure levels ranging from 49.1 to $73.4 \mathrm{~dB}(\mathrm{~A})$.

Finally, all sounds were shortened to a uniform duration of $20 \mathrm{~s}$, and next to the three original recordings of road traffic noise, 12 additional stimuli for the listening experiment were constructed by combining the original ones with different fountain and bird sounds. For each road traffic noise excerpt, two fountain sounds were selected based on the signal-to-noise ratio (SNR): one with an $L_{\mathrm{Aeq}}$ of $10-15 \mathrm{~dB}(\mathrm{~A})$ lower than that of the road traffic noise and one with an $L_{\mathrm{Aeq}}$ of $0-4.5 \mathrm{~dB}(\mathrm{~A})$ lower, as suggested by earlier research. ${ }^{3,5}$ For constructing the stimuli with bird sound, the same procedure was followed, except for the cases of major and minor road traffic noise, in which the level of birds was about $11 \mathrm{~dB}(\mathrm{~A})$ higher than that of the road traffic, for the loudest bird sound excerpts. This was done in order to achieve realistic levels of bird sound. Consequently, the bird sound was still audible during the vehicle passages. The 15 resulting stimuli had A-weighted sound pressure levels ranging from 59.6 to 73.8 $\mathrm{dB}(\mathrm{A})$, with no significant level differences between both ears. Further acoustical measures of the stimuli are listed in Table 1. The number of stimuli formed a compromise between having a wide variation in sound combinations and staying within the limits of what is possible to assess using a paired comparison test.

\subsection{Apparatus and tasks}

During the experiment, subjects were seated in a sound attenuating chamber. Sounds were presented through a closed-type headphone, and the presentation and response collection were controlled by a computer program (a set of MATLAB scripts with graphical user interface). The playback equipment was calibrated beforehand using the HATS system. All instruction and tests were conducted in Dutch.

The experiment consisted of two parts: a paired comparison test followed by a semantic differential scaling test (always in this order). For each trial of the paired comparison test, participants were presented two stimuli, to which they could listen as many times as needed. After having listened at least once to both, they had to select the stimulus in which the road traffic noise sounded the loudest. Due to time constraints and limits caused by sensory fatigue, it was not feasible to present each of the $n(n-1) / 2=105$ possible comparisons to each subject. Therefore, a randomized design was followed in which each subject was presented a sequence of 30 randomly selected pairs. Subsequently, in the semantic differential scaling test, all stimuli were presented once, in random order. The participants had to scale the overall stimulus according to two semantic differentials: unpleasant/pleasant and uneventful/eventful. For this purpose, the participants could shift two sliders in the user interface, with both opposing terms on either side.

\subsection{Data analysis}

The data of the paired comparison test are analyzed according to the Bradley-TerryLuce (BTL) probabilistic choice model. ${ }^{8,9}$ The BTL model assumes that choice probabilities are related to an underlying ratio-scale:

$$
p_{a b}=\frac{v(a)}{v(a)+v(b)},
$$

in which $p_{a b}$ denotes the probability of selecting object $a$ in a paired comparison involving objects $a$ and $b$, and in which $v(a)$ and $v(b)$ are the ratio-scale values of these objects (unique up to multiplication with a constant). The latter can be estimated from the choice frequencies of the paired comparison test, using a maximum likelihood 
Table 2. Cumulative paired comparison matrix. Absolute frequencies are given with which the road traffic noise of the stimulus in the row was judged to be louder than the road traffic noise of the stimulus in the column.

\begin{tabular}{lrrrrrrrrrrrrrrr}
\hline \hline \multicolumn{1}{c}{ FwO } & FwB1 & FwB2 & FwF1 & FwF2 & MaO & MaB1 & MaB2 & MaF1 & MaF2 & MiO & MiB1 & MiB2 & MiF1 & MiF2 \\
\hline FwO & - & 25 & 21 & 22 & 20 & 14 & 14 & 17 & 18 & 21 & 15 & 21 & 17 & 26 & 30 \\
FwB1 & 22 & - & 20 & 15 & 9 & 21 & 21 & 20 & 21 & 21 & 18 & 15 & 18 & 16 & 23 \\
FwB2 & 9 & 12 & - & 30 & 7 & 12 & 12 & 6 & 14 & 18 & 20 & 31 & 20 & 19 & 15 \\
FwF1 & 12 & 12 & 24 & - & 22 & 9 & 16 & 14 & 16 & 14 & 22 & 30 & 19 & 23 & 24 \\
FwF2 & 9 & 10 & 11 & 21 & - & 28 & 15 & 20 & 15 & 20 & 14 & 16 & 16 & 11 & 18 \\
MaO & 12 & 9 & 7 & 8 & 22 & - & 18 & 10 & 20 & 11 & 19 & 27 & 14 & 20 & 9 \\
MaB1 & 9 & 18 & 5 & 8 & 10 & 19 & - & 20 & 6 & 11 & 21 & 14 & 26 & 25 & 21 \\
MaB2 & 5 & 11 & 8 & 14 & 11 & 10 & 22 & - & 19 & 17 & 19 & 13 & 16 & 21 & 19 \\
MaF1 & 14 & 11 & 5 & 6 & 17 & 12 & 7 & 15 & - & 16 & 16 & 12 & 9 & 18 & 10 \\
MaF2 & 9 & 13 & 7 & 7 & 1 & 11 & 8 & 7 & 21 & - & 15 & 12 & 17 & 18 & 14 \\
MiO & 6 & 6 & 5 & 9 & 8 & 8 & 9 & 9 & 15 & 22 & - & 22 & 13 & 13 & 13 \\
MiB1 & 4 & 9 & 5 & 7 & 6 & 11 & 3 & 8 & 11 & 15 & 17 & - & 16 & 15 & 11 \\
MiB2 & 7 & 8 & 4 & 11 & 9 & 7 & 11 & 6 & 7 & 18 & 16 & 23 & - & 22 & 16 \\
MiF1 & 2 & 6 & 11 & 7 & 9 & 9 & 16 & 5 & 6 & 21 & 17 & 18 & 23 & - & 19 \\
MiF2 & 14 & 6 & 12 & 12 & 7 & 12 & 12 & 15 & 10 & 9 & 10 & 12 & 14 & 26 & - \\
\hline \hline
\end{tabular}

method. ${ }^{8}$ The estimation procedure has been generalized for cases in which not all pairs are presented an equal number of times, ${ }^{10}$ as is the case in this paper.

The BTL model is very restrictive, as it assumes that test participants make choices consistently according to a single underlying scale, which may not hold for any given dataset; the appropriateness of a model inferred from choice frequencies can be checked using a likelihood ratio test. ${ }^{8}$ Although more widely used in economics and sociology, the BTL model has already been used in a number of studies in the field of auditory perception, e.g., for determining a ratio-scale of unpleasantness of various environmental sounds. ${ }^{11}$ Recently, a MATLAB script has been developed for performing the scale value estimation and for testing the validity of the model for the given dataset. $^{12}$

\section{Results}

\subsection{Paired comparison test}

The individual choice datasets were inspected for consistency before further processing. This revealed a median number of 1 (range 0-5) circular triad (for which $a>b$ and $b>c$ but $a<c$ ) per individual. Note that each subject only made 30 out of 105 possible comparisons, selected randomly. Therefore, the maximum possible number of circular triads varied between subjects (from 22 to 29 with median 26). It was found that the actual number of circular triads was significantly below the number to be expected by chance alone, for all subjects $\left(\chi^{2}\right.$-test, $\left.\alpha=0.05\right)$. Subsequently, the individual choice frequencies were pooled across the 100 subjects, resulting in the cumulative paired comparison matrix given in Table 2. The ratio-scale values that best predict the pooled choice probabilities were numerically estimated using a maximum likelihood procedure. ${ }^{12}$ Performing a likelihood ratio test showed that the BTL model describes the present paired comparison data quite well; the null hypothesis assuming the validity of the model may not be rejected $\left[\chi^{2}(91)=92.53 ; p=0.436\right]$. Consequently, the BTL ratio-scale values, normalized such that the lowest value is 1.0 , represent the common underlying road traffic noise loudness scale that was used by the participants; values are given in Table 1.

Figure 1(a) shows the BTL scale values and 95\% confidence intervals for the loudness of the traffic sound for each of the stimuli. To investigate in which conditions 

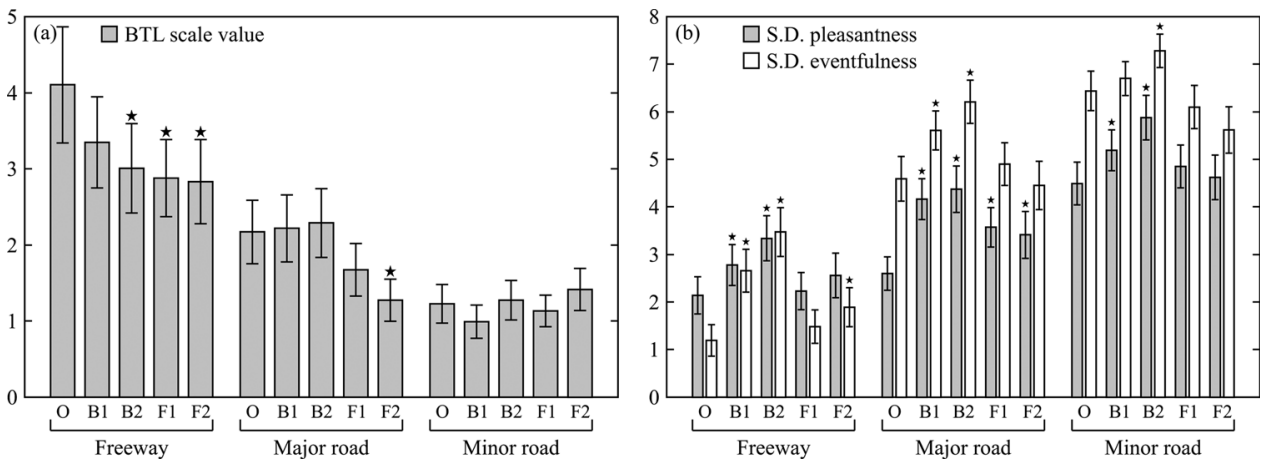

Fig. 1. (a) Estimated BTL scale values for the loudness of the traffic sound (arbitrary units), based on a total of 30 (paired comparisons) $\times 100$ (participants) $=3000$ judgments; (b) average pleasantness and eventfulness for the semantic differential (SD) test, based on 100 judgments for each stimulus. The error bars denote the $95 \%$ confidence intervals; the stars denote significant loudness reductions or pleasantness/eventfulness improvements $(\alpha=0: 05)$ as compared to the baseline stimulus (labeled "O").

the addition of fountain or bird sound resulted in a significant reduction in loudness, as compared to the respective baseline stimulus with only traffic noise (labeled "O"), a series of $t$-tests were performed; significant reductions $(\alpha=0.05)$ are marked with a star. Visual inspection of Fig. 1(a) shows that the addition of fountain sound reduced the loudness of the freeway noise significantly, for both considered fountain sound levels and the loudness of the major road traffic noise, but only for the higher fountain sound level. No significant effects are found for the loudness of the minor road traffic noise. The addition of bird sound reduced the loudness of the freeway noise significantly, but only for the higher bird sound level.

\subsection{Semantic differential test}

The last two columns in Table 1 show the results of the semantic differential scaling test, on a scale from 0 (not at all pleasant/eventful) to 10 (very pleasant/eventful), averaged over all participants; Fig. 1(b) shows the results graphically. To investigate in which conditions the addition of fountain or bird sound resulted in a significant improvement in soundscape pleasantness or eventfulness, as compared to the resp. baseline stimulus with only traffic noise (labeled "O"), a series of paired $t$-tests were performed; significant increases $(\alpha=0.05)$ are again marked with a star. On the one hand, visual inspection of Fig.1(b) shows that the addition of fountain sound only improved soundscape pleasantness for the major road traffic noise and soundscape eventfulness for the freeway noise (for the fountain sound with higher relative level). On the other hand, the addition of bird sound improved soundscape pleasantness and eventfulness for all cases considered, except for the eventfulness of the minor road traffic noise using the bird sound with lower relative sound level.

\section{Discussion and conclusions}

The results of the paired comparison test showed that adding fountain sound to soundscapes dominated by road traffic noise may reduce the loudness of the latter, which agrees with earlier findings. ${ }^{1}$ However, significant results were only found for freeway and major road traffic noise, i.e., those cases in which the traffic noise has low temporal variability. Adding bird sound had the same effect only for the freeway noise; surprisingly in the other cases, the bird sound had a higher SNR. One possible explanation (in contrast to earlier explanations based on target-masker confusion ${ }^{1}$ ) is that auditory attention is drawn to that sound of the mixture which has the highest temporal variability in sound level, and that the resulting lower amount of attention paid to the other sounds leads to a reduction in perceived loudness. In the present study, the loudness of the fountain and bird sounds was not assessed, so it was not possible to 
check if the results are symmetric in the type of sound. It has to be noted that the fountain sound stimuli used in the present study, and consequently their temporal and spectral characteristics, differ from those used in the study by Nilsson et al. ${ }^{\mathrm{P}}$ and that also the subjective evaluation method used here is different. As is noted by Nilsson et al., ${ }^{1}$ adding wanted sounds may reduce the loudness of unwanted sounds, but to the price of increasing overall sound levels, which could decrease the soundscape quality. The results of the semantic differential scaling test showed that, at least for the sound levels considered in this work, adding fountain sound only improved soundscape pleasantness significantly for the major road traffic noise situation, but not for the (stationary) freeway noise or the minor road traffic noise, which contrasts earlier findings in which similar relative sound levels were considered. ${ }^{3,5}$ On the other hand, adding the sound of birds did result in a significant increase in soundscape pleasantness and eventfulness in almost all investigated conditions. These findings suggest that, in order to effectively achieve a soundscape quality improvement, acoustic design measures will have to address more than only the loudness of unwanted sounds, as soundscape quality is influenced heavily by the meaning associated with the different sounds that are heard.

\section{Acknowledgments}

B.D.C is a postdoctoral fellow of the Research Foundation-Flanders (FWO-Vlaanderen); the support of this organization is gratefully acknowledged.

\section{References and links}

${ }^{1}$ M. E. Nilsson, J. Alvarsson, M. Rådsten-Ekman, and K. Bolin, "Auditory masking of wanted and unwanted sounds in a city park,” Noise Control Eng. J. 58, 524-531 (2010).

${ }^{2}$ K. Bolin, M. E. Nilsson, and S. Khan, "The potential of natural sounds to mask wind turbine noise," Acta Acust. Acust. 96, 131-137 (2010).

${ }^{3}$ J. Y. Jeon, P. J. Lee, J. You, and J. Kang, "Perceptual assessment of quality of urban soundscapes with combined noise sources and water sounds," J. Acoust. Soc. Am. 127, 1357-1366 (2010).

${ }^{4}$ G. R. Watts, R. J. Pheasant, K. V. Horoshenkov, and L. Ragonesi, "Measurement and subjective assessment of water generated sounds," Acta Acust. Acust. 95, 1032-1039 (2009).

${ }^{5}$ J. You, P. J. Lee, and J. Y. Jeon, "Evaluating water sounds to improve the soundscape of urban areas affected by traffic noise," Noise Control Eng. J. 58, 477-483 (2010).

${ }^{6}$ B. De Coensel, A. Bockstael, L. Dekoninck, D. Botteldooren, B. Schulte-Fortkamp, J. Kang, and M. E. Nilsson, "The soundscape approach for early stage urban planning: A case study," in Proceedings of Internoise (Lisbon, Portugal, 2010), p. 504.

${ }^{7}$ N. Durlach, "Auditory masking: Need for improved conceptual structure," J. Acoust. Soc. Am. 120, 1787-1790 (2006).

${ }^{8}$ R. A. Bradley and M. E. Terry, "Rank analysis of incomplete block designs: I. The method of paired comparisons," Biometrika 39, 324-345 (1952).

${ }^{9}$ R. D. Luce, Individual Choice Behavior: A Theoretical Analysis (Wiley, New York, 1959).

${ }^{10} \mathrm{O}$. Dykstra, Jr., "Rank analysis of incomplete block designs: A method of paired comparisons employing unequal repetitions on pairs," Biometrics 16, 176-188 (1960).

${ }^{11}$ W. Ellermeier, M. Mader, and P. Daniel, "Scaling the unpleasantness of sounds according to the BTL model: Ratio-scale representation and psychoacoustical analysis," Acta Acust. Acust. 90, 101-107 (2004).

${ }^{12} \mathrm{~F}$. Wickelmaier and C. Schmid, "A Matlab function to estimate choice model parameters from pairedcomparison data,” Behav. Res. Methods Instrum. Comput. 36, 29-40 (2004). 